\title{
PROPAGATION OF THE SCALE PROPERTY USING GAMES
}

\author{
ITAY NEEMAN
}

The aim of this short paper is to introduce the reader to the notion of a scale and to some of the basic techniques involved in the propagation of the scale property through the use of infinite games. None of the results presented is due to the author. For a full history see Moschovakis [2]. We work throughout the paper with the space $\omega^{\omega}$. For $s \in \omega^{<\omega}$ we use $N_{s}$ to denote the set $\left\{x \in \omega^{\omega} \mid x\right.$ extends $s\}$. The sets $N_{s}, s \in \omega^{<\omega}$, form the basic open subsets of $\omega^{\omega}$. Following standard abuse we refer to the space $\omega^{\omega}$, equipped with the topology generated by these basic open set, as $\mathbb{R}$.

Given a set $A \subset \mathbb{R}$ let $G(A)$ denote the following game: Players I and II alternate playing $x(n)$ for $n \in \omega$ subject to the order displayed in Diagram 1, with $x(n) \in \omega$ for each $n$. If, after $\omega$ moves, the real $x=\langle x(n) \mid n<\omega\rangle$ belongs to $A$ then player I wins. Otherwise player II wins. $G(A)$ is determined if one of the two players has a winning strategy in the game.

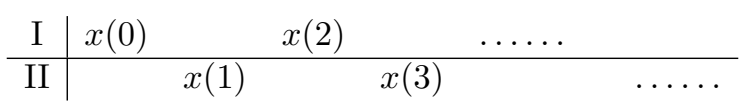

Diagram 1. The game $G(A)$.

For $B \subset \mathbb{R} \times \mathbb{R}$ and $x \in \mathbb{R}$ let $B_{x}=\{y \in \mathbb{R} \mid\langle x, y\rangle \in B\}$. This is the $x$-section of $B$. Define $\partial B$ to be the set $\{x \in \mathbb{R} \mid$ player I has a winning strategy in $\left.G\left(B_{x}\right)\right\}$. We sometimes write $(\partial y) B(x, y)$, or $(\partial y)\langle x, y\rangle \in B$, for the statement $x \in \partial B$. This is deliberately meant to conjure up the notation used for statements involving the quantifiers $(\forall y)$ and $(\exists y)$. ( $\partial y)$ really is a quantifier, giving precise meaning to the chain $(\exists y(0))(\forall y(1))(\exists(y(2)) \cdots \cdots$ of quantifiers over $\omega$.

Let $B \subset \mathbb{R} \times \mathbb{R}$ be open. Note that for each $\langle x, y\rangle \in B$ there exists some $n<\omega$ so that $N_{x\lceil n} \times N_{y\lceil n} \subset B$. Let $n(x, y)$ denote the least such $n$. We refer to $n(x, y)$ as the time of entry of $\langle x, y\rangle$ into $B$. For $\langle x, y\rangle \notin B$ we set $n(x, y)=\omega$.

Let $A=\partial B$. For $x, x^{*} \in \mathbb{R}$ define $H\left(x^{*}, x\right)$ to be the following game: Players "first" and "second" (denoted F and S respectively) alternate moves subject to the format in Diagram 2. The moves are played sequentially from left to right, and are presented in two separate lines only for future convenience. The letters $\mathrm{F}$ and $\mathrm{S}$ indicate which player is responsible for each move. Each of the moves is a natural number. An infinite run leading to reals $y=\langle y(i) \mid i<\omega\rangle$ and

Supported by the National Science Foundation under Grant No. DMS-0094174. 


$$
\begin{array}{llllllll}
\mathrm{F} y(0) & & & \mathrm{S} y(1) & \mathrm{F} y(2) & & & \\
& \mathrm{S} y^{*}(0) & \mathrm{F} y^{*}(1) & & & \mathrm{S} y^{*}(2) & \ldots
\end{array}
$$

Diagram 2. The game $H\left(x^{*}, x\right)$.

$y^{*}=\left\langle y^{*}(i) \mid i<\omega\right\rangle$ is won by $\mathrm{S}$ if

$$
\langle x, y\rangle \notin B, \quad \text { or } \quad\langle x, y\rangle \in B \wedge\left\langle x^{*}, y^{*}\right\rangle \in B \wedge n\left(x^{*}, y^{*}\right) \leq n(x, y) .
$$

Otherwise the run is won by $\mathrm{F}$. With our convention that $n(x, y)=\omega$ for $\langle x, y\rangle \notin$ $B$, a run $\left\langle y^{*}, y\right\rangle$ of $H\left(x^{*}, x\right)$ is won by $\mathrm{S}$ just in case that $n\left(x^{*}, y^{*}\right) \leq n(x, y)$.

$H\left(x^{*}, x\right)$ thus involves a simultaneous play of both $G\left(B_{x}\right)$, taking place on the upper line in Diagram 2, and $G\left(B_{x^{*}}\right)$, taking place on the lower line. We think of the former as owned by F, and of the latter as owned by S. Each plays for I on the line she owns, and for II on the line owned by her opponent.

To win, S must make sure that the play on her line (namely the lower line, where she plays for I) does not lag behind the play on her opponent's line: if the play on her opponent's line enters $B$ then she has to make sure that the play on her line enters $B$, at the same time or earlier.

Define a relation $\preceq$ on $\mathbb{R}$ by setting $x^{*} \preceq x$ iff $\mathrm{S}$ has a winning strategy in $H\left(x^{*}, x\right)$. This winning strategy should be viewed as a "translation mechanism." It translates a strategy for I in $G\left(B_{x}\right)$ into a strategy for I in $G\left(B_{x^{*}}\right)$, making sure that the translated strategy never lags behind the original strategy.

ClaIM 1. The relation $\preceq$ is reflexive.

Proof. S can win $H(x, x)$ simply by copying the moves played by $\mathrm{F}$. More precisely, the strategy defined by the conditions $y^{*}(n)=y(n)$ for even $n$ and $y(n)=y^{*}(n)$ for odd $n$ is winning for $\mathrm{S}$ in $H(x, x)$.

Claim 2. The relation $\preceq$ is transitive.

Proof. Suppose that $x^{* *} \preceq x^{*} \preceq x$. Let $\tau_{1}$ be a winning strategy for $\mathrm{S}$ in $H\left(x^{*}, x\right)$ and let $\tau_{2}$ be a winning strategy for $\mathrm{S}$ in $H\left(x^{* *}, x^{*}\right)$. Let $\tau$ be the strategy in $H\left(x^{* *}, x\right)$ obtained by composing $\tau_{1}$ and $\tau_{2}$. A typical play according to $\tau$ is illustrated in Diagram 3. The play starts in the upper left corner with a move by $\mathrm{F}$, and proceeds along the arrows obtaining additional moves through plays by $\mathrm{F}$ in $H\left(x^{* *}, x\right)$, uses of $\tau_{1}$, and uses of $\tau_{2}$, as indicated in the diagram. Note that a play $\left\langle y^{* *}, y\right\rangle$ is according to $\tau$ iff there is a real $y^{*}$ so that $\left\langle y^{* *}, y^{*}\right\rangle$ is according to $\tau_{2}$ and $\left\langle y^{*}, y\right\rangle$ is according to $\tau_{1}$. It is easy using this characterization to check that $\tau$ is winning for $\mathrm{S}$ in $H\left(x^{* *}, x\right)$.

Lemma 3. For $x, x^{*} \in \mathbb{R}$ set $x \prec x^{*}$ iff $x^{*} \npreceq x$. Suppose that each of the games $H\left(x^{*}, x\right), x, x^{*} \in \mathbb{R}$, is determined. Then the relation $\prec$ is wellfounded.

Proof. Suppose for contradiction that $\left\langle x_{i}\right| i\langle\omega\rangle$ is a sequence of reals so that $x_{i+1} \prec x_{i}$, meaning $x_{i} \npreceq x_{i+1}$, for each $i$. Using the assumption of determinacy it follows that $\mathrm{F}$ has a winning strategy in $H\left(x_{i}, x_{i+1}\right)$. Let $\tau_{i}$ be such a strategy. Construct reals $y_{i}, i<\omega$, following Diagram 4 . The construction proceeds column by column from left to right, setting $y_{0}(k)=0$ for each even 


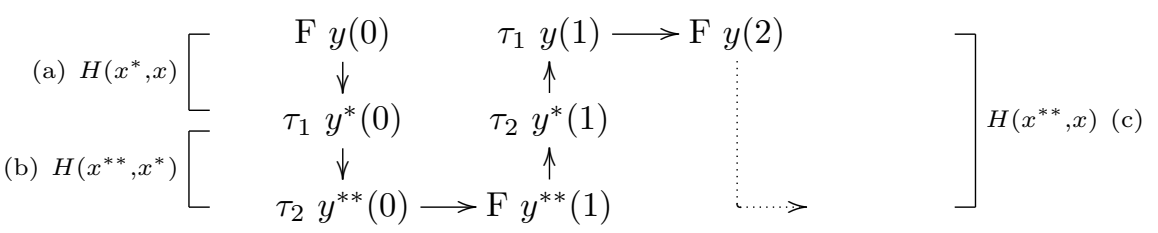

Diagram 3. Composing $\tau_{1}$ and $\tau_{2}$.

$k$ and using the strategies $\tau_{i}$, as indicated in the diagram, to produce all other objects.

For each $i<\omega$, the lines corresponding to $y_{i}$ and $y_{i+1}$ together form a play of $H\left(x_{i}, x_{i+1}\right)$, according to $\tau_{i}$. (For $i=1$ the progress of this play is indicated in the diagram through squiggly arrows, and the play itself is indicated in boldface.) Using the fact that $\tau_{i}$ is winning for $\mathrm{F}$ in $H\left(x_{i}, x_{i+1}\right)$ it follows that $\left\langle x_{i+1}, y_{i+1}\right\rangle \in$ $B$ and that $n\left(x_{i+1}, y_{i+1}\right)<n\left(x_{i}, y_{i}\right)$ for each $i<\omega$. But this gives an infinite descending sequence of natural numbers, and hence a contradiction.

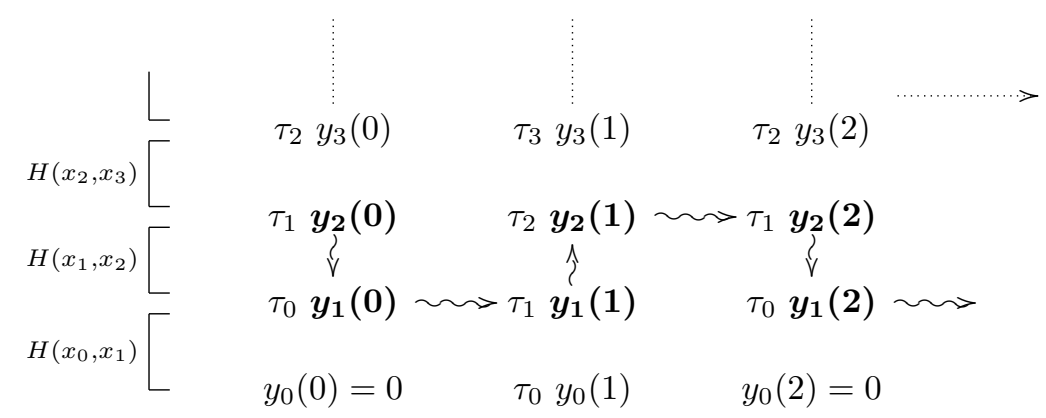

Diagram 4. The wellfoundedness of $\prec$.

REMARK 4 . It follows from the last lemma that $\preceq$ is total: if $x \npreceq x^{*}$ and $x^{*} \npreceq$ $x$ then $\left\langle x, x^{*}, x, x^{*}, \ldots\right\rangle$ is an infinite descending sequence in $\prec$, a contradiction.

We know now that the relation $\preceq$ is a prewellorder, meaning that the relation $x \sim y$ iff $x \preceq y \wedge y \preceq x$ is an equivalence relation and that $\preceq$ induces a wellordering of the equivalence classes of $\sim$. Let $\varphi: \mathbb{R} \rightarrow$ ON be the rank function associated to $\preceq$. Precisely, $\varphi$ is defined through the condition $\varphi(x)=\sup \{\varphi(\bar{x})+1 \mid \bar{x} \prec x\}$ (with the supremum of the empty set taken to be 0 ). We shall see that the relationship between $A$ and $x \mapsto \varphi(x)$ is analogous to the relationship between the open set $B$ and the function $\langle x, y\rangle \mapsto n(x, y)$ defined earlier.

Note that

$$
\text { if }\langle x, y\rangle \in B \text { and } n\left(x^{*}, y^{*}\right) \leq n(x, y) \text { then }\left\langle x^{*}, y^{*}\right\rangle \in B \text {. }
$$

We now establish a similar relationship between the set $A=\partial B$ and the function $x \mapsto \varphi(x)$ :

Lemma 5. Suppose that $x \in A$ and $\varphi\left(x^{*}\right) \leq \varphi(x)$. Then $x^{*} \in A$. 
Proof. Since $x \in A=\partial B$, player I has a winning strategy in $G\left(B_{x}\right)$. Let $\sigma$ be such a strategy. Since $\varphi\left(x^{*}\right) \leq \varphi(x)$, S has a winning strategy in $H\left(x^{*}, x\right)$. Let $\tau$ be such a strategy. $\sigma$ and $\tau$ combine naturally to give rise to a strategy $\sigma^{*}$ for player I in $G\left(B_{x^{*}}\right) . \sigma^{*}$ is characterized by the condition that $y^{*}$ is according to $\sigma^{*}$ iff there is a real $y$ so that $y$ is according to $\sigma$ and $\left\langle y^{*}, y\right\rangle$ is according to $\tau$.

A typical run $y^{*}$ of $\sigma^{*}$ is presented on the lower line of Diagram 5 , and the associated real $y$ is presented on the upper line. Since $\sigma$ is winning for I in $G\left(B_{x}\right)$ the real $y$ must belong to $B_{x}$. In other words $\langle x, y\rangle \in B$. Since $\tau$ is winning for $\mathrm{S}$ in $H\left(x^{*}, x\right), n\left(x^{*}, y^{*}\right) \leq n(x, y)$. By $(1)$ above $\left\langle x^{*}, y^{*}\right\rangle \in B$. This shows that $\sigma^{*}$ is winning for I in $G\left(B_{x^{*}}\right)$, and hence $x^{*} \in A$.

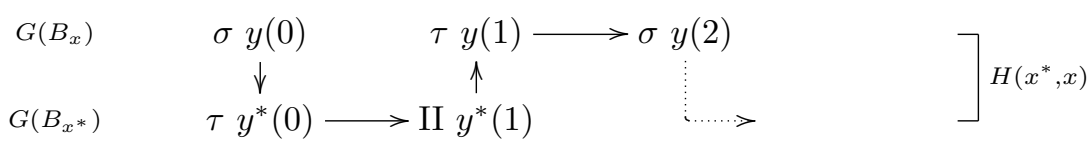

Diagram 5. Composing $\sigma$ and $\tau$.

The last lemma shows that $A$ forms an initial segment of $\mathbb{R}$ in the prewellorder given by $\varphi$, just as $B$ forms an initial segment of $\mathbb{R} \times \mathbb{R}$ in the prewellorder given by $\langle x, y\rangle \mapsto n(x, y)$. Note that the complement of $B$ is a single equivalence class at the very top of the prewellorder given by $\langle x, y\rangle \mapsto n(x, y)$. Precisely,

$$
\text { if }\langle x, y\rangle \notin B \text { then } n\left(x^{*}, y^{*}\right) \leq n(x, y) \text { for all }\left\langle x^{*}, y^{*}\right\rangle \in \mathbb{R} \times \mathbb{R} \text {. }
$$

The next claim establishes the same property for $\varphi$ and $A$.

Claim 6. Suppose that each of the games $G\left(B_{x}\right), x \in \mathbb{R}$, is determined. Let $x, x^{*} \in \mathbb{R}$, and suppose that $x \notin A$. Then $x^{*} \preceq x$.

Proof. Since $x \notin A=\partial B$, and $G\left(B_{x}\right)$ is determined, player II must have a winning strategy in $G\left(B_{x}\right)$. Let $\sigma$ be such a strategy. Let $\tau$ be the strategy for $\mathrm{S}$ in $H\left(x^{*}, x\right)$ which follows $\sigma$ on the upper line, and plays 0s on the lower line. Plays $\left\langle y^{*}, y\right\rangle$ according to $\tau$ are characterized by the condition that $y$ is according to $\sigma$ and $y^{*}(k)=0$ for each even $k$. From the first clause and the fact that $\sigma$ is winning for II in $G\left(B_{x}\right)$ is follows that $\langle x, y\rangle \notin B$, so that $\left\langle y^{*}, y\right\rangle$ is won by $\mathrm{S}$ in $H\left(x^{*}, x\right)$.

Recall that $B \subset \mathbb{R}^{2}$ is open, that is $\boldsymbol{\Sigma}_{1}^{0}$, and $n(x, y)$ is equal to the least $n$ so that $N_{x \mid n} \times N_{y \mid n} \subset B$ if $\langle x, y\rangle \in B$, and to $\omega$ if $\langle x, y\rangle \in \mathbb{R}^{2}-B$. Define $\sqsubseteq$ by setting $\left\langle x^{*}, y^{*}\right\rangle \sqsubseteq\langle x, y\rangle$ iff $n\left(x^{*}, y^{*}\right) \leq n(x, y)$, and define $\sqsubset$ by setting $\left\langle x^{*}, y^{*}\right\rangle \sqsubset\langle x, y\rangle$ iff $n\left(x^{*}, y^{*}\right)<n(x, y)$. Both are relations on $\mathbb{R}^{2}$, equivalently subsets of $\mathbb{R}^{2} \times \mathbb{R}^{2}$. It is easy to see that

$$
\text { both } \sqsubset \text { and } \sqsubseteq \cap\left(B \times \mathbb{R}^{2}\right) \text { are } \boldsymbol{\Sigma}_{1}^{0} \text {. }
$$

By $\sqsubseteq \cap\left(B \times \mathbb{R}^{2}\right)$ we mean the set $\{\langle\bar{x}, \bar{y}, x, y\rangle \mid n(\bar{x}, \bar{y}) \leq n(x, y) \wedge\langle\bar{x}, \bar{y}\rangle \in B\}$. The restriction to $\langle\bar{x}, \bar{y}\rangle \in B$ is important. The full relation $\sqsubseteq$ is not $\boldsymbol{\Sigma}_{1}^{0}$. (We could, for symmetry, also restrict $\sqsubset$ to $B \times \mathbb{R}^{2}$ in (3). But this would not make any difference: $\langle\bar{x}, \bar{y}\rangle \sqsubset\langle x, y\rangle$ already implies that $\langle\bar{x}, \bar{y}\rangle \in B$.) 
For a pointclass ${ }^{1} \Gamma$ let $\partial \Gamma$ be the pointclass $\{\partial D \mid D \in \Gamma\}$. The set $A=\circlearrowright B$ is $\partial \Sigma_{1}^{0}$. The next lemma establishes the parallel of property (3) for $A$ and $\varphi$.

Lemma 7. Suppose that all length $\omega$ games with $\boldsymbol{\Sigma}_{1}^{0}$ payoff are determined. Then $\prec$ and $\preceq \cap(A \times \mathbb{R})$ are both $\partial \boldsymbol{\Sigma}_{1}^{0}$.

Proof. Using determinacy, $\bar{x} \prec x$ iff $\mathrm{F}$ has a winning strategy in $H(x, \bar{x})$. A run $\langle y, \bar{y}\rangle$ of $H(x, \bar{x})$ is won by $\mathrm{F}$ iff $n(\bar{x}, \bar{y})<n(x, y)$, and this is a $\boldsymbol{\Sigma}_{1}^{0}$ condition by property (3). Hence the set $\{\langle\bar{x}, x\rangle \mid \mathrm{F}$ has a winning strategy in $H(x, \bar{x})\}$ is $\partial \boldsymbol{\Sigma}_{1}^{0}$.

It remains to prove that $\preceq \cap(A \times \mathbb{R})$ is $\partial \boldsymbol{\Sigma}_{1}^{0}$.

Define $H^{\prime}\left(x^{*}, x\right)$ to be played according to the rules of $H\left(x^{*}, x\right)$ but with the modified payoff condition that $\mathrm{S}$ wins just in case that $\left\langle x^{*}, y^{*}\right\rangle \in B$ and $n\left(x^{*}, y^{*}\right) \leq n(x, y)$. Note that this modified condition is $\boldsymbol{\Sigma}_{1}^{0}$ by property (3). Hence:

(i) The set $\left\{\left\langle x^{*}, x\right\rangle \mid \mathrm{S}\right.$ has a winning strategy in $\left.H^{\prime}\left(x^{*}, x\right)\right\}$ is $\partial \mathbf{\Sigma}_{1}^{0}$.

Note further that the modified condition is harder for $\mathrm{S}$ than the original condition. Hence:

(ii) If $\mathrm{S}$ has a winning strategy in $H^{\prime}\left(x^{*}, x\right)$ then she also has a winning strategy in $H\left(x^{*}, x\right)$.

We intend to show that for $x^{*} \in A$ the games are in fact equivalent.

Claim 8. Let $x^{*} \in A$. Then $\mathrm{S}$ has a winning strategy in $H^{\prime}\left(x^{*}, x^{*}\right)$.

Proof. Suppose for contradiction that $\mathrm{S}$ does not have a winning strategy in $H^{\prime}\left(x^{*}, x^{*}\right)$. By determinacy then $\mathrm{F}$ has a winning strategy. Let $\tau$ be such a strategy.

Let $\sigma$ be a winning strategy for I in $G\left(B_{x^{*}}\right)$. Player I has a winning strategy in this game since $x^{*}$ is assumed to be in $A$.

Diagram 6 shows how to compose $\sigma$ and infinitely many copies of $\tau$ to produce a sequence of reals $y_{i}, i<\omega$, with the property that $y_{0}$ is according to $\sigma$, and for each $i,\left\langle y_{i}, y_{i+1}\right\rangle$ is according to $\tau$. (Each copy of $H^{\prime}\left(x^{*}, x^{*}\right)$ in the diagram is labelled by a roman letter, and the copies of $\tau$ have superscripts indicating which of the copies of $H^{\prime}\left(x^{*}, x^{*}\right)$ they belong to. The copy of $H^{\prime}\left(x^{*}, x^{*}\right)$ labelled (b) is highlighted in squiggly arrows in the diagram, and the moves by $\mathrm{F}$ in this game, made by the copy of $\tau$ labelled (b), are indicated in boldface.)

Since $\sigma$ is winning for $\mathrm{I}$ in $G\left(B_{x^{*}}\right),\left\langle x^{*}, y_{0}\right\rangle \in B$. Since $\tau$ is winning for $\mathrm{F}$ in $H^{\prime}\left(x^{*}, x^{*}\right)$, either $\left\langle x^{*}, y_{i}\right\rangle \notin B$ or $n\left(x^{*}, y_{i}\right) \not \leq n\left(x^{*}, y_{i+1}\right)$, for each $i<\omega$. Notice that the latter disjunct implies that $\left\langle x^{*}, y_{i+1}\right\rangle \in B$, by property (2). It therefore follows by induction that $\left\langle x^{*}, y_{i}\right\rangle \in B$ and $n\left(x^{*}, y_{i+1}\right)<n\left(x^{*}, y_{i}\right)$ for each $i<\omega$. But this gives an infinite descending sequence of natural numbers, a contradiction.

Claim 9. Suppose that $\mathrm{S}$ has a winning strategy in $H\left(x^{*}, x\right)$ and that $x^{*} \in A$. Then $\mathrm{S}$ has a winning strategy in $H^{\prime}\left(x^{*}, x\right)$.

\footnotetext{
${ }^{1}$ By a pointclass we always mean a class of sets closed under recursive substitutions, conjunctions, disjunctions, and bounded number quantifications.
} 


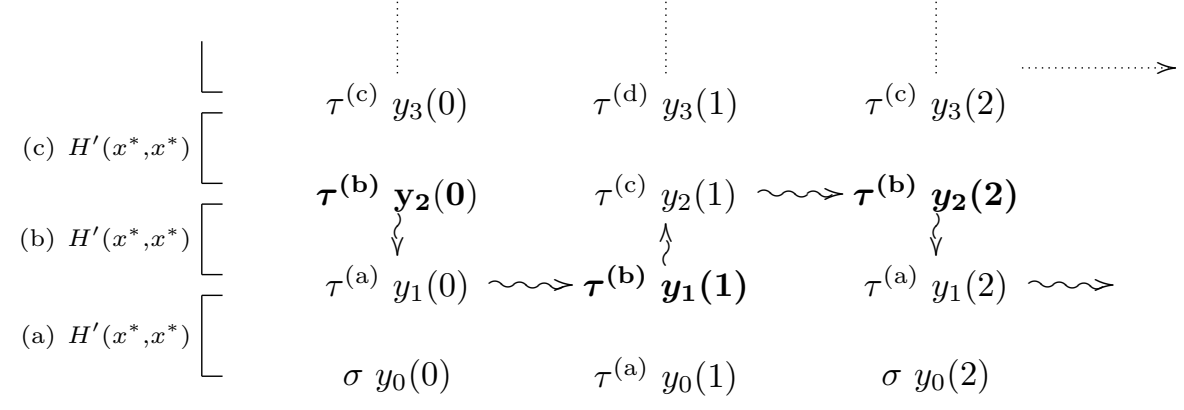

Diagram 6. F cannot have a winning strategy in $H^{\prime}\left(x^{*}, x^{*}\right)$ for $x^{*} \in A$.

Proof. Let $\tau_{1}$ be a winning strategy for $\mathrm{S}$ in $H\left(x^{*}, x\right)$, and using the previous claim let $\tau_{2}$ be a winning strategy for $\mathrm{S}$ in $H^{\prime}\left(x^{*}, x^{*}\right)$. Let $\tau$ be obtained by composing $\tau_{1}$ and $\tau_{2}$ in the manner of Diagram 3. (But here the games labelled (b) and (c) in the diagram are $H^{\prime}\left(x^{*}, x^{*}\right)$ and $H^{\prime}\left(x^{*}, x\right)$ respectively.) Then $\tau$ is winning for $\mathrm{S}$ in $H^{\prime}\left(x^{*}, x\right)$.

From the last claim and condition (ii) above it follows that for $x^{*} \in A$, S has a winning strategy in $H\left(x^{*}, x\right)$ iff she has a winning strategy in $H^{\prime}\left(x^{*}, x\right)$. From this and condition (i) it follows that the set $\left\{\left\langle x^{*}, x\right\rangle \mid x^{*} \in A\right.$ and $\mathrm{S}$ has a winning strategy in $\left.H\left(x^{*}, x\right)\right\}$ is $\partial \boldsymbol{\Sigma}_{1}^{0}$.

LIST 10. The following list summarizes the properties of $\varphi$ and $A$ obtained so far (with $\Gamma$ standing for $\partial \boldsymbol{\Sigma}_{1}^{0}$ ):

(1) If $x \in A$ and $\varphi(\bar{x}) \leq \varphi(x)$ then $\bar{x} \in A$.

(2) If $x \notin A$ and $\bar{x} \notin A$ then $\varphi(x)=\varphi(\bar{x})$.

(3) Both the sets $\{\langle\bar{x}, x\rangle \mid \varphi(\bar{x})<\varphi(x)\}$ and $\{\langle\bar{x}, x\rangle \mid \bar{x} \in A \wedge \varphi(\bar{x}) \leq \varphi(x)\}$ are in $\Gamma$.

Conditions (1) and (2) merely note that $\mathbb{R}-A$ forms a single equivalence class of the prewellorder induced by $\varphi$, located above all elements of $A$ in this prewellorder. Notice that any function $\varphi: A \rightarrow \mathrm{ON}$ can be extended to a function on $\mathbb{R}$ satisfying conditions (1) and (2) simply by setting $\varphi(x)=\sup \{\varphi(\bar{x})+1 \mid$ $\bar{x} \in A\}$ for $x \in \mathbb{R}-A$. We use the same letter to refer both to the function defined on $A$ and to an extension of the function to $\mathbb{R}$ subject to conditions (1) and (2).

Condition (3) in List 10 is the crucial one, connecting $\varphi$ to the pointclass $\Gamma$. The next definition abstracts an equivalent condition, that refers only to the restriction of $\varphi$ to $A$. The equivalence is proved in Claim 12 .

Definition 11. A function $\varphi: A \rightarrow \mathrm{ON}$ is a $\Gamma$ norm on $A$ just in case that there are sets $U$ and $V$ in $\Gamma$ and $\neg \Gamma$ respectively, so that, for every $x \in A$,

$$
\{\bar{x} \mid \varphi(\bar{x}) \leq \varphi(x)\}=\{\bar{x} \mid\langle\bar{x}, x\rangle \in U\}=\{\bar{x} \mid\langle\bar{x}, x\rangle \in V\} .
$$

Claim 12. Let $\varphi: A \rightarrow \mathrm{ON}$ and extend $\varphi$ to $\mathbb{R}$ in line with conditions (1) and (2) in List 10. Then $\varphi$ is a $\Gamma$ norm iff it satisfies condition (3) in the list. 
Proof. Assuming condition (3), let $U=\{\langle\bar{x}, x\rangle \mid \bar{x} \in A \wedge \varphi(\bar{x}) \leq \varphi(x)\}$, and let $V=\{\langle\bar{x}, x\rangle \mid \varphi(x) \nless \varphi(\bar{x})\}$. Assuming the condition in Definition 11 note that $\varphi(\bar{x})<\varphi(x)$ iff $\bar{x} \in A \wedge\langle x, \bar{x}\rangle \notin V$, and that $\bar{x} \in A \wedge \varphi(\bar{x}) \leq \varphi(x)$ iff $(x \in A \wedge\langle\bar{x}, x\rangle \in U) \vee(\bar{x} \in A \wedge\langle x, \bar{x}\rangle \notin V)$.

The condition in Definition 11 states that for each $x \in A$, the initial segment $\{\bar{x} \mid \varphi(\bar{x}) \leq \varphi(x)\}$ belongs to both $\Gamma(x)$ and $\neg \Gamma(x)$, and that this holds uniformly in $x$. Working with norms in this paper it is more convenient to use the equivalent characterization in List 10, and we shall do this without further comment throughout the paper.

A pointclass $\Gamma$ is said to have the prewellordering property if every set in $\Gamma$ admits a $\Gamma$ norm. The prewellordering property has various applications to questions in descriptive set theory, see for example the theorem on reduction in $[1, \S 2]$.

The sequence of results given above shows how to produce a $\partial \boldsymbol{\Sigma}_{1}^{0}$ norm on a given $\partial \boldsymbol{\Sigma}_{1}^{0}$ set, starting from $\boldsymbol{\Sigma}_{1}^{0}$ norms on $\boldsymbol{\Sigma}_{1}^{0}$ sets. But there is nothing specific to $\boldsymbol{\Sigma}_{1}^{0}$ sets in any of the proofs. They generalize routinely to yield the following theorem:

THEOREM 13. Let $\Gamma$ be a pointclass. Suppose that every length $\omega$ game with payoff in $\Gamma$ is determined. Suppose that $\Gamma$ has the prewellordering property. Then Ә厂 has the prewellordering property.

Proof. Let $B \subset \mathbb{R}^{2}$ belong to $\Gamma$ and let $\theta: \mathbb{R} \rightarrow$ ON be a $\Gamma$ norm on $B$. Follow the sequence of definitions and claims above, only replacing the uses of $n(x, y)$ by uses of $\theta(x, y)$. It is easy to check that the proofs adapt, showing that the resulting function $\varphi$ is a $\partial \Gamma$ norm on $A=\partial B$. Let us only note that the definability expressed by condition (3) in List 10, for the norm $\theta$, is such that all the games that come up in the adapted proofs have payoff sets in $\Gamma$, and are therefore determined. (Their determinacy is needed in the proofs.)

There is one crucial property of the norm $\langle x, y\rangle \mapsto n(x, y)$ that was not considered in the discussion so far. It is easy to check that this norm and the $\boldsymbol{\Sigma}_{1}^{0}$ set $B$ satisfy

let $\left\langle x_{i}, y_{i}\right\rangle, i<\omega$, be elements of $B$. Suppose that $\lim _{i \rightarrow \infty} x_{i}$ and $\lim _{i} \longrightarrow \infty y_{i}$ exist and let $x_{\infty}$ and $y_{\infty}$ respectively denote the limits. Suppose that $n\left(x_{i}, y_{i}\right)$ is eventually constant as $i \longrightarrow \infty$. Then $\left\langle x_{\infty}, y_{\infty}\right\rangle \in B$ and $n\left(x_{\infty}, y_{\infty}\right) \leq$ eventual value of $n\left(x_{i}, y_{i}\right)$.

As stated this additional property is not true at the level of $\partial \boldsymbol{\Sigma}_{1}^{0}$ sets, but we can obtain a parallel property at that level by using countably many norms.

Given $x, x^{*} \in \mathbb{R}, p \in \omega^{<\omega}$ of even length, say $2 k$, and $h, h^{*} \in \omega$, define $H_{p}\left(x^{*}, h^{*}, x, h\right)$ to be played as follows: Players $\mathrm{F}$ and $\mathrm{S}$ alternate moves subject to the format in Diagram 7 . The moves are played sequentially from left to right, starting from the vertical line, and each of the moves is a natural number. At the end of an infinite run we set $y=p^{-}\langle h\rangle \frown\langle y(i) \mid 2 k<i<\omega\rangle$ and $y^{*}=p^{\frown}\left\langle h^{*}\right\rangle \frown\left\langle y^{*}(i) \mid 2 k<i<\omega\right\rangle$. The run is won by $\mathrm{S}$ if

$$
\langle x, y\rangle \notin B, \quad \text { or }\langle x, y\rangle \in B \wedge\left\langle x^{*}, y^{*}\right\rangle \in B \wedge n\left(x^{*}, y^{*}\right) \leq n(x, y),
$$


and otherwise the run is won by F.

$$
\begin{array}{llll}
p(0) & \cdots & p(2 k-1) \\
p(0) & \cdots & p(2 k-1)
\end{array} \quad h\left(\begin{array}{lllll} 
& & \mathrm{S} y(2 k+1) & \mathrm{F} y(2 k+2) & \\
\mathrm{F} y^{*}(2 k+1) & & & \ldots
\end{array}\right.
$$

Diagram 7. The game $H_{p}\left(x^{*}, h^{*}, x, h\right)$.

$H_{p}\left(x^{*}, h^{*}, x, h\right)$ may thus be viewed as a version of $H\left(x^{*}, x\right)$ with $y\lceil 2 k+1$ set equal to $p^{\frown}\langle h\rangle$ and $y^{*} \mid 2 k+1$ set equal to $p^{\frown}\left\langle h^{*}\right\rangle$. For reason of notational convenience we refer to $\left\langle y^{*}, y\right\rangle$, rather than the sequence of actual moves, as a run of $H_{p}\left(x^{*}, h^{*}, x, h\right)$.

Define $\preceq_{p}$ by setting $\left\langle x^{*}, h^{*}\right\rangle \preceq_{p}\langle x, h\rangle$ iff $\mathrm{S}$ has a winning strategy in the game $H_{p}\left(x^{*}, h^{*}, x, h\right)$. The previous proofs adapt to show that $\preceq_{p}$ is a prewellorder. Let $\varphi_{p}: \mathbb{R} \times \omega \rightarrow \mathrm{ON}$ be the associated rank function, defined by $\varphi_{p}(x, h)=$ $\sup \left\{\varphi_{p}(\bar{x}, \bar{h})+1 \mid\langle\bar{x}, \bar{h}\rangle \prec_{p}\langle x, h\rangle\right\} . \varphi_{p}$ is then a $\partial \boldsymbol{\Sigma}_{1}^{0}$ norm on the set $\{\langle x, h\rangle \mid$ $p \frown\langle h\rangle$ is a winning position for I in $\left.G\left(B_{x}\right)\right\}$.

For $p \in \omega^{<\omega}$ of even length and $x \in \mathbb{R}$ let $\psi_{p}(x)=\min \left\{\varphi_{p}(x, h) \mid h \in \omega\right\}$ and let $h_{p}(x)$ be the smallest number $h$ realizing the minimum, that is $h_{p}(x)=$ $\min \left\{h \mid \varphi_{p}(x, h)=\psi_{p}(x)\right\}$.

EXERCISE 14. Let $x \in A$. Let $p \in \omega^{<\omega}$ be a node of even length, say $2 k$. Suppose that $p(2 i)=h_{p \nmid 2 i}(x)$ for each $i<k$. Show that $p$ is a winning position for I in $G\left(B_{x}\right)$.

HiNT FOR THE CASE $k=1$. Let $\sigma$ be a winning strategy for I in $G\left(B_{x}\right)$. Let $h$ be the first move played by $\sigma$. By assumption $p(0)=h_{\emptyset}(x)$, and it follows from the definition of $h_{\emptyset}(x)$ that $\varphi_{\emptyset}(x, p(0)) \leq \varphi(x, h)$. So S has a winning strategy, $\tau$ say, in the game $H_{\emptyset}(x, p(0), x, h)$. Diagram 8 shows how to win $G\left(B_{x}\right)$ from $p$, against an opponent who plays for II, using a composition of $\sigma$ and $\tau$.

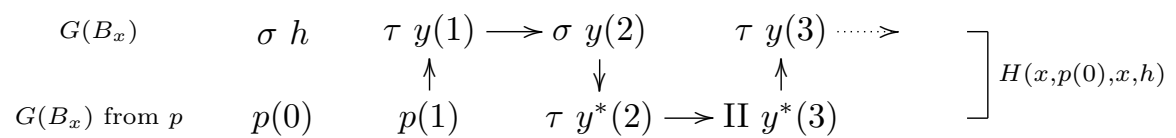

Diagram 8. Hint for Exercise 14.

Claim 15. The norm $\psi_{\emptyset}$ is equivalent to the earlier norm $\varphi$ on $A$, in the sense that $\psi_{\emptyset}\left(x^{*}\right) \leq \psi_{\emptyset}(x)$ iff $\varphi\left(x^{*}\right) \leq \varphi(x)$.

Proof. $\psi_{\emptyset}\left(x^{*}\right) \leq \psi_{\emptyset}(x)$ iff $(\forall n)\left(\exists n^{*}\right)$ so that $\mathrm{S}$ has a winning strategy in $H_{\emptyset}\left(x^{*}, n^{*}, x, n\right)$. Prepending moves corresponding to the quantifier string $(\forall n)\left(\exists n^{*}\right)$ to the game $H_{\emptyset}\left(x^{*}, n^{*}, x, n\right)$ we obtain precisely the game $H\left(x^{*}, x\right)$. So $\psi_{\emptyset}\left(x^{*}\right) \leq \psi_{\emptyset}(x)$ iff $\mathrm{S}$ has a winning strategy in $H\left(x^{*}, x\right)$ iff $\varphi\left(x^{*}\right) \leq \varphi(x)$. $\dashv$

EXERCISE 16. Let $A_{p}=\left\{x \mid p\right.$ is a winning position for I in $\left.G\left(B_{x}\right)\right\}$. Show that $\psi_{p}$ is a $\partial \boldsymbol{\Sigma}_{1}^{0}$ norm on $A_{p}$. In fact, show that the sets $\left\{\langle p, \bar{x}, x\rangle \mid \psi_{p}(\bar{x})<\right.$ $\left.\psi_{p}(x)\right\}$ and $\left\{\langle p, \bar{x}, x\rangle \mid \bar{x} \in A_{p} \wedge \psi_{p}(\bar{x}) \leq \psi_{p}(x)\right\}$ are in $\partial \boldsymbol{\Sigma}_{1}^{0}$. 
Hint. That $\left\{\langle p, \bar{x}, x\rangle \mid \psi_{p}(\bar{x})<\psi_{p}(x)\right\}$ is $\partial \boldsymbol{\Sigma}_{1}^{0}$ follows directly from (determinacy and) the definitions. For $\left\{\langle p, \bar{x}, x\rangle \mid \bar{x} \in A_{p} \wedge \psi_{p}(\bar{x}) \leq \psi_{p}(x)\right\}$ use an argument similar to that in the proof of Lemma 7.

We now approach the parallel of property (4):

Theorem 17. Let $\left\langle x_{i} \mid i<\omega\right\rangle$ be a sequence of reals in the set $A=\partial B$. Suppose that $\lim _{i} \longrightarrow \infty x_{i}$ exists and let $x_{\infty}$ denote this limit. Suppose that for each $p$, both $\psi_{p}\left(x_{i}\right)$ and $h_{p}\left(x_{i}\right)$ are eventually constant as $i \longrightarrow \infty$. Let $\lambda_{p}$ and $h_{p}$ respectively be their eventual values. Then:

(1) $x_{\infty}$ belongs to $A$.

(2) For each $p,\left\langle\psi_{p}\left(x_{\infty}\right), h_{p}\left(x_{\infty}\right)\right\rangle \leq_{\mathrm{Lex}}\left\langle\lambda_{p}, h_{p}\right\rangle$.

Proof. We show that $\varphi_{\emptyset}\left(x_{\infty}, h_{\emptyset}\right) \leq \lambda_{\emptyset}$. A similar proof establishes that $\varphi_{p}\left(x_{\infty}, h_{p}\right) \leq \lambda_{p}$ for each $p$. Condition (2) of the theorem follows directly from this. Condition (1) follows from the instance $\psi_{\emptyset}\left(x_{\infty}\right) \leq \psi_{\emptyset}\left(x_{i}\right)$ (for all sufficiently large $i$ ) using Lemma 5 and Claim 15.

For each $p$ let $k(p)<\omega$ be large enough that $\psi_{p}\left(x_{i}\right)=\lambda_{p}$ and $h_{p}\left(x_{i}\right)=h_{p}$ for all $i \geq k(p)$. Choose $k(p)$ inductively so that $k(p)>k(\bar{p})$ whenever $\bar{p}$ is a strict initial segment of $p$.

Let $p_{0}=\emptyset$ and let $k_{0}=k(\emptyset)$.

Suppose for contradiction that $\varphi_{\emptyset}\left(x_{\infty}, h_{\emptyset}\right) \not \leq \lambda_{\emptyset}$. Since $\lambda_{\emptyset}=\psi_{\emptyset}\left(x_{k_{0}}\right)=$ $\min \left\{\varphi_{\emptyset}\left(x_{k_{0}}, h\right) \mid h \in \omega\right\}$ this means that there is some $h \in \omega$ so that $\varphi_{\emptyset}\left(x_{\infty}, h_{\emptyset}\right) \not$ $\varphi_{\emptyset}\left(x_{k_{0}}, h\right)$. Fix such an $h$.

Using determinacy the fact that $\varphi_{\emptyset}\left(x_{\infty}, h_{\emptyset}\right) \not \leq \varphi_{\emptyset}\left(x_{k_{0}}, h\right)$ implies that $\mathrm{F}$ has a winning strategy in the game $H_{\emptyset}\left(x_{\infty}, h_{\emptyset}, x_{k_{0}}, h\right)$ which we denote $H^{(\infty)}$. Fix such a winning strategy $\sigma$.

Now construct sequences $\left\{k_{n}\right\},\left\{p_{n}\right\},\left\{\tau^{(n)}\right\}$, and $\left\{y_{n}\right\}$ so that:

(a) $p_{0} \subset p_{1} \subset p_{2} \cdots$ and $\operatorname{lh}\left(p_{n}\right)=2 n$ for each $n$. Let $y_{\infty}=\bigcup_{n<\omega} p_{n}$.

(b) $y_{0}(0)=h, y_{1}(0)=h_{\emptyset}$, and the pair $\left\langle y_{\infty}, y_{0}\right\rangle$ is a run of $H^{(\infty)}$ played according to $\sigma$.

(c) $p_{n+1}=p_{n} \frown\left\langle h_{p_{n}}, y_{n+1}(2 n+1)\right\rangle$.

(d) $k_{n+1}=k\left(p_{n+1}\right)$.

(e) $\tau^{(n)}$ is a winning strategy for $\mathrm{S}$ in the game $H_{p_{n}}\left(x_{k_{n+1}}, h_{p_{n}}, x_{k_{n}}, y_{n}(2 n)\right)$ which we denote $H^{(n)}$.

(f) The pair $\left\langle y_{n+1}, y_{n}\right\rangle$ is a run of $H^{(n)}$ played according to $\tau^{(n)}$.

Diagram 9 illustrates the construction. The construction begins on the upper left corner, with the assignments $y_{0}(0)=h$ and $y_{1}(0)=h_{\emptyset}$. The construction continues following the arrows in the diagram, assigning to each entry a value either by setting it equal to $h_{p_{n}}$ for some $n$ or by using one of the strategies, as indicated. The symbol " in an entry indicates copying the value of the entry above it.

Note that the values of $y_{n+1}(2 n)$ (set equal to $\left.h_{p_{n}}\right)$ and $y_{n+1}(2 n+1)$ (determined using $\sigma$ ) can be determined before $k_{n+1}$ is known. (They do not depend on $\tau^{(n)}$.) Once these assignments are made we set $p_{n+1}=p_{n} \frown\left\langle y_{n+1}(2 n), y_{n+1}(2 n+\right.$ $1)\rangle$, and set $k_{n+1}=k\left(p_{n+1}\right)$. Since $y_{n+1}(2 n)=h_{p_{n}}$, and since both $k_{n}$ and $k_{n+1}$ 
are greater than or equal to $k\left(p_{n}\right)$,

$$
\begin{aligned}
\varphi_{p_{n}}\left(x_{k_{n+1}}, y_{n+1}(2 n)\right) & =\lambda_{p_{n}} \\
& =\varphi_{p_{n}}\left(x_{k_{n}}, h_{p_{n}}\right) \\
& \leq \varphi_{p_{n}}\left(x_{k_{n}}, y_{n}(2 n)\right)
\end{aligned}
$$

Thus $\mathrm{S}$ has a winning strategy in $H^{(n)}=H_{p_{n}}\left(x_{k_{n+1}}, y_{n+1}(2 n), x_{k_{n}}, y_{n}(2 n)\right)$, allowing us to pick $\tau^{(n)}$ subject to condition (e) above and continue with the construction, following the arrows.

$y_{\infty}$ (whose entries are indicated in boldface in Diagram 9) and $y_{0}$ together form a run of $H_{\emptyset}\left(x_{\infty}, h_{\emptyset}, x_{k_{0}}, h\right)$, played according to $\sigma$. Since $\sigma$ is winning for $\mathrm{F}$ in the game,

(i) $n\left(x_{k_{0}}, y_{0}\right)<n\left(x_{\infty}, y_{\infty}\right)$.

$y_{n+1}$ and $y_{n}$ together form a run of $H^{(n)}$ according to $\tau^{(n)}$. Since $\tau^{(n)}$ is winning for $\mathrm{S}$ in the game,

(ii) $n\left(x_{k_{n+1}}, y_{n+1}\right) \leq n\left(x_{k_{n}}, y_{n}\right)$.

It follows from this, and the wellfoundedness of $\omega$, that $n\left(x_{k_{n}}, y_{n}\right)$ is eventually constant as $n \longrightarrow \infty$. Now $\lim _{n \longrightarrow \infty} x_{k_{n}}=x_{\infty}$ by the assumptions of the theorem, and $\lim _{n} \longrightarrow \infty y_{n}=y_{\infty}$ by construction. Using property (4) above it follows that

(iii) $n\left(x_{\infty}, y_{\infty}\right) \leq$ the eventual value of $n\left(x_{k_{n}}, y_{n}\right)$.

But from this and condition (ii) it follows that $n\left(x_{\infty}, y_{\infty}\right) \leq n\left(x_{k_{0}}, y_{0}\right)$, and this contradicts condition (i).

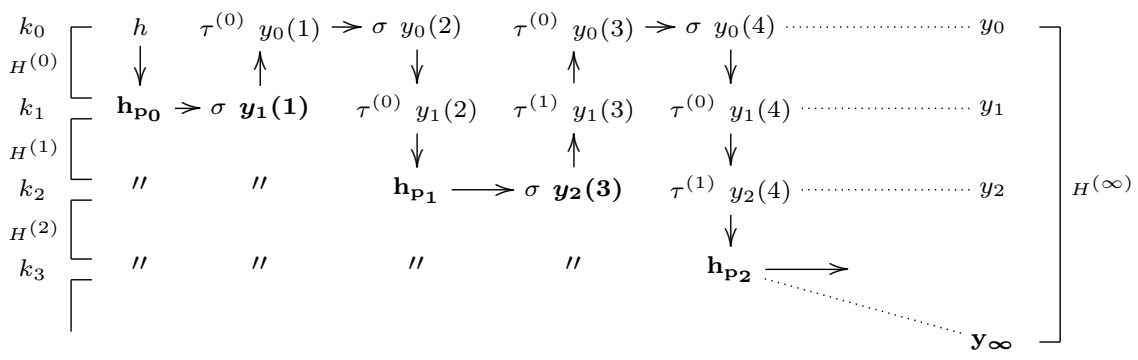

Diagram 9. The proof of Theorem 17

Definition 18. Let $A \subset \mathbb{R}$. A countable sequence $\left\{\theta_{l}\right\}$ of norms on $A$ is called a scale on $A$ if it satisfies the following limit condition:

(*) Let $x_{i}$ for $i<\omega$ belong to $A$. Suppose that $\lim _{i} \longrightarrow \infty x_{i}$ exists and let $x_{\infty}$ denote the limit. Suppose that for each $l, \theta_{l}\left(x_{i}\right)$ is eventually constant as $i \longrightarrow \infty$, and let $\lambda_{l}$ denote the eventual value. Then: $x_{\infty} \in A$; and for each $l, \theta_{l}\left(x_{\infty}\right) \leq \lambda_{l}$.

$\left\{\theta_{l}\right\}$ is called a $\Gamma$ scale if both the sets $\left\{\langle l, \bar{x}, x\rangle \mid \theta_{l}(\bar{x})<\theta_{l}(x)\right\}$ and $\{\langle l, \bar{x}, x\rangle \mid$ $\left.\bar{x} \in A \wedge \theta_{l}(\bar{x}) \leq \theta_{l}(x)\right\}$ belong to $\Gamma$, or, equivalently, if there are sets $U$ and $V$ in $\Gamma$ and $\neg \Gamma$ respectively so that

$$
\left\{\bar{x} \mid \theta_{l}(\bar{x}) \leq \theta_{l}(x)\right\}=\{\bar{x} \mid\langle\bar{x}, x, l\rangle \in U\}=\{\bar{x} \mid\langle\bar{x}, x, l\rangle \in V\}
$$


for all $l<\omega$ and all $x \in A$.

$\Gamma$ has the scale property if each set in $\Gamma$ admits a $\Gamma$ scale.

REMARK 19. Many applications involve scales $\left\{\theta_{l}\right\}$ so that $\left\{\langle\bar{x}, x\rangle \mid \theta_{l}(\bar{x})<\right.$ $\left.\theta_{l}(x)\right\}$ and $\left\{\langle\bar{x}, x\rangle \mid \bar{x} \in E \wedge \theta_{l}(\bar{x}) \leq \theta_{l}(x)\right\}$ belong to $\Gamma$ for each individual $l$, meaning that each $\theta_{l}$ is a $\Gamma$ norm, but the joins $\left\{\langle l, \bar{x}, x\rangle \mid \theta_{l}(\bar{x})<\theta_{l}(x)\right\}$ and $\left\{\langle l, \bar{x}, x\rangle \mid \bar{x} \in A \wedge \theta_{l}(\bar{x}) \leq \theta_{l}(x)\right\}$ do not belong to $\Gamma$. We call such scales weakly $\Gamma$, and say that $\Gamma$ is weakly scaled if every set in $\Gamma$ admits a weakly $\Gamma$ scale.

Theorem 17 shows that $\left\{\psi_{p}, h_{p}\right\}$ is a scale on $A=\partial B$. But it need not be a $\partial \Sigma_{1}^{0}$ scale. The problem is with the shift from $A$ to $A_{p}$ in the definability of the norms $\psi_{p}$ in Exercise 16. We now solve this problem by restricting to $p$ which are winning for I in $G\left(B_{x}\right)$, through a use of Exercise 14.

Call $p \in \omega^{<\omega}$ of even length $2 k$ correct for $x \in A$ just in case that $(\forall i<k)$ $p(2 i)=h_{p \nmid 2 i}(x)$.

REMARK 20. The assumption in Theorem 17, that $\psi_{p}\left(x_{i}\right)$ and $h_{p}\left(x_{i}\right)$ are eventually constant as $i \longrightarrow \infty$ for each $p$, can be weakened to apply only to $p$ which are correct for $x_{i}$ for almost all $i$ (meaning all but finitely many $i$ ), as these are the only $p$ which come up during the proof of the theorem.

Note that for every $r \in \omega^{<\omega}$ of length $k$ there is a unique $p$ which is correct for $x$ and so that $(\forall i<k) p(2 i+1)=r(i)$. Let

$$
\vec{\alpha}_{r}(x)=\left\langle\psi_{p \nmid 0}(x), h_{p \nmid 0}(x), \psi_{p \nmid 2}(x), h_{p \nmid 2}(x), \ldots, \psi_{p}(x), h_{p}(x)\right\rangle
$$

for this unique $p$.

Set $\bar{x} \unlhd_{r} x$ iff $x \notin A$ or $\bar{x} \in A \wedge x \in A \wedge \vec{\alpha}_{r}(\bar{x}) \leq_{\text {Lex }} \vec{\alpha}_{r}(x)$. It is clear that $\unlhd_{r}$ is a prewellorder on $\mathbb{R}$. Let $\psi_{r}^{\prime}: \mathbb{R} \rightarrow \mathrm{ON}$ be its rank function.

Claim 21. The relations $\left\{\langle r, \bar{x}, x\rangle \mid \psi_{r}^{\prime}(\bar{x})<\psi_{r}^{\prime}(x)\right\}$ and $\{\langle r, \bar{x}, x\rangle \mid \bar{x} \in A \wedge$ $\left.\psi_{r}^{\prime}(\bar{x}) \leq \psi_{r}^{\prime}(x)\right\}$ are both $\circlearrowright \boldsymbol{\Sigma}_{1}^{0}$.

Proof. This is a simple calculation using Exercise 16, a parallel of the same exercise for the norm $\varphi_{p}$, and the fact that if $p$ is correct for $x$ then $x \in A_{p}$, given by Exercise 14 .

Claim 22. Let $x_{i}$ be a sequence of reals in $A$ and suppose that for each $r$, $\psi_{r}^{\prime}\left(x_{i}\right)$ is eventually constant as $i \longrightarrow \infty$. Suppose that $p$ is correct for $x_{i}$ for almost all $i$. Then $\psi_{p}\left(x_{i}\right)$ and $h_{p}\left(x_{i}\right)$ are eventually constant as $i \longrightarrow \infty$.

Proof. Let $k$ be such that $\operatorname{lh}(p)=2 k$. Let $r=\langle p(1), \ldots, p(2 k-1)\rangle$. Let $n$ be large enough that $p$ is correct for $x_{i}$ for all $i>n$. Then $\vec{\alpha}_{r}\left(x_{i}\right)=$ $\left\langle\psi_{p \nmid 0}\left(x_{i}\right), h_{p \nmid 0}\left(x_{i}\right), \psi_{p \nmid 2}\left(x_{i}\right), h_{p \nmid 2}\left(x_{i}\right), \ldots, \psi_{p}\left(x_{i}\right), h_{p}\left(x_{i}\right)\right\rangle$ for all $i>n$, and the fact that $\psi_{p}\left(x_{i}\right)$ and $h_{p}\left(x_{i}\right)$ are eventually constant follows from the fact that $\vec{\alpha}_{r}\left(x_{i}\right)$ is eventually constant.

Corollary 23. The sequence $\left\{\psi_{r}^{\prime}\right\}_{r \in \omega}<\omega$ is a $\partial \boldsymbol{\Sigma}_{1}^{0}$ scale on $A=\partial B$.

Proof. The definability required by Definition 18 is given by Claim 21 . The limit condition is given by Theorem 17 using Remark 20 and Claim 22 .

Our work so far produced a $\partial \boldsymbol{\Sigma}_{1}^{0}$ scale on a given $\partial \boldsymbol{\Sigma}_{1}^{0}$ set, starting from a norm satisfying property (4) above. But in fact the argument can be adapted to start with a scale, rather than a single norm, and to use the limit condition in Definition 18, rather than property (4). The result is the following theorem: 
Theorem 24. Let $\Gamma$ be a pointclass. Let $B \subset \mathbb{R}^{2}$ belong to $\Gamma$. Let $\left\{\theta_{l}\right\}_{l<\omega}$ be a $\Gamma$ scale (respectively weakly $\Gamma$ scale) on $B$. Suppose that $\Gamma$ determinacy holds. Define $H_{p}, \preceq_{p}, \varphi_{p}, h_{p}, \psi_{p}$, and $\psi_{r}^{\prime}$ as above, but replacing the condition " $n\left(x^{*}, y^{*}\right) \leq n(x, y)$ " with the condition

$$
\left\langle\theta_{0}\left(x^{*}\right), \ldots, \theta_{\operatorname{lh}(p) / 2}\left(x^{*}\right)\right\rangle \leq_{\operatorname{Lex}}\left\langle\theta_{0}(x), \ldots, \theta_{\operatorname{lh}(p) / 2}(x)\right\rangle
$$

throughout.

Then $\left\{\psi_{r}^{\prime}\right\}_{r \in \omega<\omega}$ is a $\partial \Gamma$ scale (respectively weakly $\partial \Gamma$ scale) on the set $\mathrm{B}$.

Proof. The proofs given above generalize routinely to these settings. Let us only make the following comments:

First, note that all the games that come up during the proofs have payoff sets in $\Gamma$, since the norms $\theta_{l}$ are all $\Gamma$ norms. The games are therefore determined. This is important since the proofs require their determinacy.

Second, condition (i) in the proof of Theorem 17 is revised in the general settings to state that

(i) $\theta_{0}\left(x_{k_{0}}, y_{0}\right)<\theta_{0}\left(x_{\infty}, y_{\infty}\right)$.

Third, condition (ii) in the proof of Theorem 17 is revised in the general settings to state that

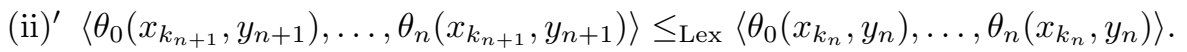

It follows from the revised condition, and from the wellfoundedness of the ordinals, that $\theta_{l}\left(x_{k_{n}}, y_{n}\right)$ is eventually constant as $n \longrightarrow \infty$, for each $l<\omega$. Using the limit condition in Definition 18 then

(iii) $)^{\prime} \theta_{l}\left(x_{\infty}, y_{\infty}\right) \leq$ eventual value of $\theta_{l}\left(x_{k_{n}}, y_{n}\right)$ as $n \longrightarrow \infty$, for each $l$.

From this and condition (ii)' it certainly follows that $\theta_{0}\left(x_{\infty}, y_{\infty}\right) \leq \theta_{0}\left(x_{k_{0}}, y_{0}\right)$, contradicting condition (i)'.

Theorems 24 and 13 are propagation theorems. They show that some desirable properties, the prewellordering property in the case of Theorem 13 and scale properties in the case of Theorem 24, propagate from a pointclass $\Gamma$ to the pointclass $\partial \Gamma$. Infinite games are central to the proofs of both theorems, and both theorems require determinacy.

We noted above that the prewellordering property can be used to settle the classical problem of reduction. The scale property too has applications to classical problems, specifically to the problem of uniformization. Roughly speaking the problem involves definably selecting elements from non-empty sets of reals. The following claim is an indication of how scales connect with such selections.

Claim 25. Let $E \subset \mathbb{R}$ be non-empty and let $\left\{\theta_{l}\right\}_{l<\omega}$ be a scale on $E$. For $x \in \mathbb{R}$ set $\vec{\alpha}(x)=\left\langle\theta_{0}(x), x(0), \theta_{1}(x), x(1), \ldots\right\rangle$. Let $\left\langle\lambda_{0}, h_{0}, \lambda_{1}, h_{1}, \ldots\right\rangle$ be the lexicographic infimum of the set $\{\vec{\alpha}(x) \mid x \in E\}$. (The infimum is characterized precisely by the condition that for each $n,\left\langle\lambda_{0}, h_{0}, \ldots, \lambda_{n-1}, h_{n-1}\right\rangle$ is the lexicographically smallest element of $\{\vec{\alpha}(x)\lceil n \mid x \in E\}$.)

Then the real $y=\left\langle h_{n} \mid n<\omega\right\rangle$ belongs to $E$.

Proof. For each $n$ pick $x_{n} \in E$ so that $\vec{\alpha}\left(x_{n}\right) \mid n=\left\langle\lambda_{0}, h_{0}, \ldots, \lambda_{n-1}, h_{n-1}\right\rangle$. Note that $x_{n} \longrightarrow \infty y$ and that $\theta_{l}\left(x_{n}\right)$ is equal to $\lambda_{l}$ for all $n>l$. By the limit condition in Definition 18 it follows that $y \in E$. 


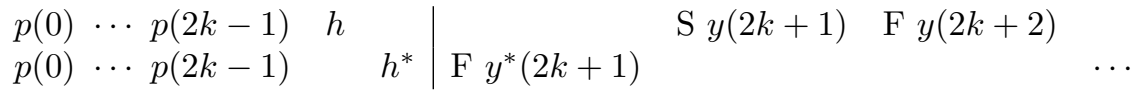

Diagram 10. The game $H_{p}\left(h^{*}, h\right)$.

Thus there is a canonical way to select an element of $E$ given a scale on $E$. This canonical selection process can be turned into a solution for the problem of uniformization, see Kechris-Moschovakis [1, §3.1].

In a similar fashion, scales can be used to select canonical winning strategies, producing $\partial \Gamma$ winning strategies in $\Gamma$ games won by the player aiming to enter the $\Gamma$ set:

EXERCISE 26. Let $E \subset \mathbb{R}$ belong to a pointclass $\Gamma$ and let $\left\{\theta_{l}\right\}_{l<\omega}$ be a $\Gamma$ scale on E. Suppose that every length $\omega$ game with payoff in $\Gamma$ is determined. Suppose that player I wins the game $G_{\omega}(E)$. Prove that player I has a winning strategy $\sigma$ which belongs to the pointclass $\partial \Gamma$, meaning that the relation ( $p$ is winning for I) $\wedge \sigma(p)=h$ is $\partial \Gamma$.

Hint. Given $p \in \omega^{<\omega}$ of even length, say $2 k$, and $h, h^{*} \in \omega$, define $H_{p}\left(h^{*}, h\right)$ to be played according to Diagram 10, with the moves played sequentially from left to right, starting from the vertical line. At the end of an infinite run set $y=p \frown\langle h\rangle \frown\langle y(i) \mid 2 k<i<\omega\rangle$ and $y^{*}=p^{\frown}\left\langle h^{*}\right\rangle \frown\left\langle y^{*}(i) \mid 2 k<i<\omega\right\rangle$. The run is won by $\mathrm{S}$ if

$$
\begin{aligned}
& y \notin E, \quad \text { or } \\
& \qquad y \in E \wedge y^{*} \in E \wedge\left\langle\theta_{0}\left(y^{*}\right), \ldots, \theta_{\operatorname{lh}(p) / 2}\left(y^{*}\right)\right\rangle \leq_{\operatorname{Lex}}\left\langle\theta_{0}(y), \ldots, \theta_{\operatorname{lh}(p) / 2}(y)\right\rangle,
\end{aligned}
$$

and otherwise the run is won by $\mathrm{F}$.

Let $Q$ be the set of positions $p \in \omega^{<\omega}$ of even length from which player I can continue to win $G_{\omega}(E)$. For $p \in Q$ and $h, h^{*} \in \omega$ set $h^{*} \preceq_{p} h$ iff S has a winning strategy in the game $H_{p}\left(h^{*}, h\right)$. Notice the similarity between the definitions here and the ones preceding Exercise 14 (with the modification indicated in Theorem 24). Adapting the proofs connected to the exercise show that:

(i) $\preceq_{p}$ is a prewellorder on $\omega$.

Let $\sigma(p)$ be the smallest number in the set $\left\{h \mid h\right.$ is $\preceq_{p}$ minimal $\}$. Show that:

(ii) The relation $p \in Q \wedge \sigma(p)=h$ is $\partial \Gamma$.

(iii) If $p$ is consistent with $\sigma$ then $p$ is a winning position for I in $G_{\omega}(E)$.

(iv) $\sigma$ is a winning strategy for I in $G_{\omega}(E)$.

Items (i) and (ii) involve adaptations of proofs preceding Exercise 14. Item (iii), which precisely parallels Exercise 14, is a warm-up for item (iv).

Claim 25 and Exercise 26 are examples of basic applications of norms and scales in descriptive set theory. The papers in Part II of this volume contain many more applications, demonstrating the fundamental importance of scales in the study of consequences of determinacy.

The papers in Part I for the most part concentrate on establishing the scale property for various pointclasses. In many cases this is done through propagation, building on and expanding the introductory methods presented here. 


\section{REFERENCES}

[1] Alexander S. Kechris and Yiannis N. Moschovakis, Notes on the theory of scales, This volume.

[2] Yiannis N. Moschovakis, Descriptive set theory, Studies in Logic and the foundations of Mathematics, vol. 100, Springer, 1980.

\section{DEPARTMENT OF MATHEMATICS \\ UNIVERSITY OF CALIFORNIA LOS ANGELES \\ LOS ANGELES, CA 90095-1555}

E-mail: ineeman@math.ucla.edu 